\title{
Desarrollo humano y desigualdad: análisis de la distribución del ingreso
}

\section{Human development and inequality: analysis of the income distribution}

\author{
Imelda Ortiz Medina ${ }^{1 *}$, Marlen Hernández Ortiz $^{1}$ and Jorge Martínez Pérez ${ }^{1}$ \\ ${ }^{1}$ Universidad Autónoma de Zacatecas, México \\ *Correspondence: imeldaortizmedina@uaz.edu.mx
}

\begin{abstract}
Resumen: Para lograr aumentar el desarrollo humano los países destinan más recursos para salud, educación, vivienda, mayores ingresos para la población, etc. En el menester de disminuir la desigualdad de ingreso, los gobiernos deben centrar sus políticas en la distribución de este. Este artículo analiza la correlación estadística y dependencia entre el Desarrollo Humano y la Desigualdad, medidos a través del Índice de Desarrollo Humano (IDH) y el Coeficiente de Gini respectivamente, para dos grupos de países con diferentes índices de desarrollo humano en los años 1990 y 2018. Una vez hecho el análisis estadístico se encontró que estas dos variables mantienen una alta correlación que es inversa, lo que significa que cuando una aumenta, la otra disminuye. También se analizó la regresión lineal simple, utilizando al IDH como variable dependiente del coeficiente de Gini, obteniendo valor de R alto, particularmente para el año 2018.
\end{abstract}

Palabras clave: desigualdad; desarrollo humano; producción; correlación bivariada; coeficiente de Pearson.

\begin{abstract}
In order to increase human development, countries allocate more resources for health, education, housing, higher income for the population, etc. In order to reduce income inequality, governments must focus their policies on the distribution of income. This article analyzes the statistical correlation and dependence between Human Development and Inequality, measured through the Human Development Index (HDI) and the Gini Coefficient respectively, for two groups of countries with different human development indices in the 1990s and 1990s. 2018. Once the statistical analysis was done, it was found that these two variables maintain a high correlation that is inverse, which means that when one increases, the other decreases. Simple linear regression was also analyzed, using the HDI as a dependent variable of the Gini coefficient, obtaining a high $\mathrm{R}$ value, particularly for the year 2018.
\end{abstract}

Keywords: inequality; human development; production; bivariate correlation; Pearson coefficient. 


\section{Introducción}

El desarrollo humano es el proceso en el cual una sociedad pretende mejorar las condiciones de vida de las personas que la conforman. Para alcanzar éste, deben aumentar aquellos bienes que lo puedan proveer tanto de las necesidades básicas como de las complementarias, implica por sí mismo: más y mejores escuelas, hospitales, servicios públicos, vivienda digna, esparcimiento, mejores salarios, etc. Por tal motivo, una de las preocupaciones centrales de la economía es el desarrollo y bienestar de los pueblos. Es decir, la economía debe buscar y encontrar un equilibrio entre crecimiento económico y desarrollo humano. Lo anterior nos lleva a preguntarnos ¿Qué es lo que un país requiere para aumentar el Desarrollo Humano? ¿En qué medida el Índice de Desarrollo Humano y la desigualdad se correlacionan? De esta forma, el propósito de este artículo es comparar la desigualdad y el desarrollo humano de un grupo de países, con diferentes niveles de desarrollo humano e identificar como se correlacionan estas dos variables. La hipótesis central es que; para alcanzar un mayor desarrollo humano es necesario distribuir el producto (ingreso nacional), es decir, es preciso disminuir la desigualdad de ingreso, por lo que ambas variables mantienen una estrecha correlación.

Este artículo es una investigación mixta de alcance exploratorio, descriptivo-correlacional y se estructura de la siguiente forma: primero están los antecedentes, enseguida se aborda la literatura relacionada con los aspectos teóricos sobre la desigualdad, el desarrollo humano y la producción (ingreso). Posteriormente, se plantea la discusión sobre la relación que guardan estas tres variables, para luego presentar el resultado del análisis de la correlación y regresión estadística entre el IDH y el Coeficiente de Gini, como uno de los principales indicadores con los cuales se mide la desigualdad.

\section{Antecedentes}

Antes de la década de los 90s la discusión sobre el tema del desarrollo puntualiza su análisis en el aumento del ingreso promedio de los países, cuya variable central era el PIB. El nivel de desarrollo humano, como advierten Bancaja e Ivie (2010), es la esencia fundamental del primer informe presentado por las Naciones Unidas, resaltando la distinción entre el Producto Interno Bruto y el Desarrollo Humano en la que se enfatiza, basados en las aportaciones de Amartya Sen (Picazzo et al., 2011), que el objetivo central del desarrollo no debía ser incrementar la riqueza material y el consumo sino proveer de oportunidades a las personas, ya que el PIB por si solo omite la problemática de los impactos medioambientales, de género, de derechos humanos o de libertades políticas y algunos aspectos más (Manfred, 2014, p. 1). Bajo esta lógica, el crecimiento del PIB solo es un medio para generar recursos y lograr el fin que es el desarrollo y bienestar de los pueblos.

Desde entonces a la fecha, los análisis de economistas y filósofos como Sen, Nussbaum, los trabajos del Programa de las Naciones Unidas para el Desarrollo (2018) entre otros, han contribuido a cambiar la forma de medir el bienestar de la sociedad, convirtiéndolo en un concepto multidimensional.

\section{Marco teórico}

\subsection{Desigualdad}

Existe una gran desigualdad social y económica entre países, derivada no necesariamente del crecimiento de la producción, sino también de los mecanismos de distribución. El Informe sobre desarrollo humano (Organización de las Naciones Unidas 2019) advierte que cualquier evaluación exhaustiva de la desigualdad debe tener en cuenta el ingreso y la riqueza. Pero, además, debe ir más allá de los dólares y las rupias para entender las diferencias existentes en 
otros aspectos del desarrollo humano y los procesos que conducen a dichas diferencias. Existe desigualdad económica, por supuesto, pero también desigualdades en facetas clave del desarrollo humano, como la salud, la educación, la dignidad y el respeto de los derechos humanos. Esas desigualdades no se manifiestan al considerar únicamente la desigualdad de ingreso y riqueza, es por eso por lo que el enfoque de la desigualdad basado en el desarrollo humano adopta una visión centrada en las personas: lo importante son las capacidades de estas para ejercer su libertad en ser y hacer aquello a lo que aspiran en la vida (Organización de las Naciones Unidas, 2019).

Ahora bien, ¿cómo medimos la distribución del ingreso o renta que se produce en cada país? Los indicadores más utilizados a la hora de medir la distribución de la renta son el Coeficiente de Gini o Índice de Gini, que indica, en un solo dato, el grado de concentración de la renta, toma valores entre cero y uno (o cien). Entre más cerca se esté del cero mayor será la distribución del ingreso, es decir menor desigualdad en la distribución de la renta. Cuanto más un país se acerque al cien, mayor será su grado de desigualdad (Lizárraga, 2013).

Existe otro índice para medir la desigualdad, el cual utiliza varios datos; la cuota de la renta nacional que es recibida por la población más pobre, la intermedia y la cuota de la renta nacional que es percibida por el $20 \%$ de la población más rica, en este índice Piketty divide a las sociedades en tres grupos (Bustos Serrano, 2018): la clase popular definida como el 50\% más bajo, la clase media, el $40 \%$ del medio, y la clase alta, que es el 10\% más alto. Así pues “La desigualdad reduce el IDH mundial en una quinta parte. Y golpea más duramente a los países de las categorías de desarrollo bajo y medio" (Organización de las Naciones Unidas, 2018, p. 3).

Si los ingresos se repartieran en proporción a la población -si el 10 por ciento inferior de la población recibiera aproximadamente el 10 por ciento de los ingresos, si el 20 por ciento inferior de la población recibiera el 20 por ciento, etcétera-, el Coeficiente de Gini sería igual a 0, no habría desigualdad. Por otra parte, si todos los ingresos fueran a parar a la persona situada en lo más alto, el Coeficiente de Gini sería igual a 1, es decir, habría una desigualdad «perfecta». Las sociedades con mayor igualdad tienen unos Coeficientes de Gini de 0,3 o menos, entre ellas están Suecia, Noruega y Alemania. Las sociedades más desiguales tienen Coeficientes de Gini de 0,5 o más, entre ellas están algunos países de África y Latinoamérica (Stiglitz, 2012).

El Producto Interno Bruto (PIB) por su parte, al medir la renta per cápita media, no informa sobre el grado de desigualdad en la distribución del ingreso (Bancaja \& Ivie, 2010, p. 3). Para el Programa de las Naciones Unidas para Desarrollo (2019b), la desigualdad en el ámbito de desarrollo humano es realmente preocupante, el acceso a la educación es muy heterogénea, mientras en un país desarrollado, más de la mitad de los jóvenes de 20 años están estudiando en el nivel superior, en los países de menor índice, pocos acceden a esta. En todos los países hay muchas personas con escasas perspectivas de vivir un futuro mejor, carecen de esperanza, sentido de propósito y dignidad; desde su situación de marginación, solo les queda contemplar a otras personas que prosperan y se enriquecen cada vez más.

El cumplimiento de los llamados derechos económicos, sociales y culturales se encuentra intrínsecamente vinculado con el bienestar tanto de aquellas personas que participan en los mercados laborales como para sus dependientes. Desvincular el cumplimiento de estos derechos con las condiciones económicas prevalecientes ha traído como consecuencia un aumento de la pobreza y la desigualdad (Reyes \& López, 2017). Una desigualdad alta puede generar consecuencias adversas para la cohesión social y la calidad de las instituciones y las políticas, lo que a su vez puede ralentizar el progreso en materia de desarrollo humano (Organización de las Naciones Unidas, 2018, p. 4).

Las desigualdades entre países están muy bien documentadas, por ejemplo, en los países con muy alto desarrollo humano se tiene una esperanza de vida de 80 años mientras que en los de bajo desarrollo humano sólo alcanza los 58.7. La escolaridad promedio va desde los 4.2 años hasta los 11.3, el PIB per cápita va desde los US\$1,585 hasta los US\$ 33,352 (Salazar \& García, 2014). Pero, además, Piketty (2020) afirma que la desigualdad no es sólo una cuestión económica 
ya que "la experiencia histórica muestra que la capacidad ideológica, política e institucional para justificar y estructurar la desigualdad en cada sociedad es lo que determina su nivel, no tanto la riqueza o el desarrollo económico en sí".

\subsection{Desarrollo humano}

Sen (2000) señala que, a pesar del incremento sin precedentes de la opulencia global, el mundo contemporáneo niega libertades elementales a enormes cantidades de personas, si no es que a la mayoría. "En suma, mayor desarrollo humano significa mayor libertad, cuya expansión implica procesos que posibilitan la libertad de acción y de elección, y que expanden las oportunidades reales de las que las personas gozan" (Organización de las Naciones Unidas 2019a, p. 30). Algunas veces la falta de libertades reales se relaciona directamente con la pobreza económica, que priva a la gente de la libertad de satisfacer el hambre, alcanzar una nutrición adecuada, obtener medicinas para enfermedades curables, contar con techo y abrigo, agua limpia e instalaciones sanitarias.

Según Sen (2000), los individuos no necesitan ser considerados los receptores pasivos de los beneficios de ingeniosos programas de desarrollo pues con oportunidades sociales adecuadas pueden construir su propio destino, para este autor, ésta es la verdadera esencia del libre y sustentable albedrío. "A los que nacen en la pobreza les resulta tan difícil huir de ella, los economistas se refieren a esa situación con el término «trampa de la pobreza»" (Stiglitz, 2012, p. 75). El IDH estima valores que van de 0 a 1, donde un valor más cercano a uno indica mayor desarrollo humano, tanto para el índice general como para sus subíndices o componentes de salud, educación e ingreso.

La construcción y difusión del enfoque de desarrollo humano ha contado con los Informes de Desarrollo Humano y el Índice de Desarrollo Humano (IDH) como herramientas principales para reorientar el debate público hacia este paradigma que pone en el centro a las personas y no las cosas (Govea, 2018), ni al mercado. Se integran dimensiones adicionales al ingreso para medir el bienestar, con base en principios como el universalismo, la sustentabilidad y la equidad (Organización de las Naciones Unidas 2019a) y otros índices compuestos para hacer un seguimiento de la pobreza, la desigualdad y el empoderamiento de las mujeres en múltiples dimensiones del desarrollo humano: el Índice de Pobreza Multidimensional (IPM), el Índice de Desarrollo Humano ajustado por la Desigualdad (IDH-D) y el Índice de Desigualdad de Género.

\subsection{Producto e Ingreso Nacional}

Derivado de la gran depresión de los años 30 del siglo XX, gobiernos de todo el mundo preocupados por los acontecimientos del momento y en aras de encontrar solución a los mismos, buscaron mecanismos para disponer de información más sistemática y ordenada sobre la producción nacional y su comportamiento y así poder diseñar políticas económicas encaminadas a evitar que esto volviese a suceder. Un estudio realizado por Bancaja e Ivie (2010) advierte que la variable más importante para este fin es el Producto Interno Bruto, el cual mide el valor de mercado de los bienes y servicios finales producidos en una sociedad durante un periodo de tiempo determinado, generalmente un año.

En aquel momento no se planteó que esa variable tuviese una relación directa con el bienestar, simplemente se afirmaba que el PIB es un instrumento que carece de juicios de valor, mide bienes y servicios finales monetizados, que al caer nos indican que hay menos inversión y, por ende, más desempleo, el cual se traduce en más pobreza, lo que se interpreta como una alerta para cualquier economía (Manfred, 2014). El PIB no fue creado como índice de bienestar social, sino sólo para cuantificar el volumen trimestral o anual de las actividades orientadas al mercado (Govea, 2018). Por lo tanto, como tal y para estos efectos es útil. Sin embargo, el PIB no computa la contribución de algunas actividades productivas esenciales para el bienestar, como es el trabajo doméstico o el cuidado de niños y ancianos en el hogar, ni tampoco contabiliza el valor del stock 
de activos de una economía, ignorando el coste del consumo o la degradación de los recursos naturales (Bancaja \& Ivie, 2010, p. 3).

Así pues, a pesar de reconocer que el PIB es la variable fundamental a la hora de hablar de crecimiento económico, no lo es cuando lo hacemos de bienestar, al incluir este último concepto, hablamos de desarrollo. Los países con alto crecimiento económico tienen más PIB, más inversión, más empleo y no necesariamente tienen mayor bienestar como mejor nivel educativo, mejores servicios de salud o mejores ingresos. El PIB puede crecer en el presente a costa del bienestar futuro, mediante la sobreexplotación de recursos naturales o deuda externa excesiva (Bancaja \& Ivie, 2010, p. 3). Para Manfred (2014), el Producto Interno Bruto no hace diferencia entre aquellas actividades que dañan la paz de los pueblos o el medio ambiente, como son la producción de drogas, el narcotráfico, la explotación de una mina a cielo abierto o la construcción de un viaducto en plena selva, entre otros. La tasa de crecimiento del PIB no tiene en cuenta los costos medio ambientales de la actividad productiva ni contabiliza la variación de la riqueza acumulada (stock), ni la degradación de los recursos naturales, ni mucho menos nos indica en manos de quien está el ingreso que se genera del aumento de la producción (Bancaja \& Ivie, 2010, p. 3).

\section{Discusión}

Manfred (2014) afirma que el PIB, al ser un agregado económico, presta poca atención a aspectos de distribución y a aquellas actividades humanas fuera de la esfera del mercado y de la valoración monetaria. Para solventar esto, diversos organismos se han planteado algunas alternativas, la más importante y conocida es la diseñada por las Naciones Unidas que es el Índice de Desarrollo Humano y diversos índices compuestos que incluyen la desigualdad social y de género. Por su parte, la Agenda 2030 y sus 17 Objetivos de Desarrollo Sostenible (ODS) reconocen el carácter multidimensional del desarrollo y la integralidad indispensable para impulsarlo. Promueven una mirada de abajo hacia arriba, con una perspectiva más focalizada en lo local, por ser este ámbito el más cercano a las personas (Organización de las Naciones Unidas, 2019b).

Más importante que el crecimiento del PIB es su redistribución. Govea $(2018$, p. 1) dice que ni el IDH ni el PIB consideran la distribución de la renta de cada país, sino que lo que se ha hecho con respecto a la distribución es calcular el PIB per cápita. Éste se calcula dividiendo el PIB entre la población total lo que tampoco resuelve el problema de fondo ya que solo es un cálculo matemático. Por otro lado, según Stiglitz (2012), los críticos de la redistribución sugieren que el costo de ésta es demasiado alto. Argumentan que podríamos tener más igualdad, pero sólo a costa de pagar el elevado precio de un crecimiento más lento y un PIB menor. No obstante, para este autor, la realidad es exactamente lo contrario pues señala que: tenemos un sistema que ha estado trabajando horas extra a fin de trasladar el dinero desde los niveles inferiores y medios hasta el nivel más alto, pero el sistema es tan ineficiente que lo que acaban ganando los de arriba es mucho menos de lo que pierden los de en medio y los de abajo. "En realidad, estamos pagando un elevado precio por nuestra creciente y desmesurada desigualdad: no solo un crecimiento más lento y un PIB menor, sino incluso más inestabilidad" (Stiglitz, 2012, p. 39).

Según el Informe sobre Desarrollo Humano (2019b), la heterogeneidad en el desarrollo humano debilita la configuración y cohesión social. A pesar de que se han logrado reducir algunos problemas estructurales relacionados con la esperanza de vida, el acceso a la educación y a la alimentación, subsisten desigualdades severas en el terreno del desarrollo humano que tienen que ver con las capacidades humanas y la libertad de los individuos para ser y hacer, aspectos determinados por las circunstancias (muchas ellas establecidas desde antes de nacer), la cultura y los valores específicos de cada país. Según Bancaja e Ivie (2010, p. 3) el PIB per cápita no mide aspectos que quedan fuera del mercado y que en los países de renta alta se valoran cada vez más (tiempo libre, hábitat, etc.). 
Stiglitz (2012) afirma que la principal falla del mercado es su ineficiencia e incapacidad de crear puestos de trabajo para todos los ciudadanos, lo que causa y acrecienta la desigualdad y no permite el desarrollo humano. Evidentemente, familias donde uno de sus miembros busca trabajo y no lo encuentra disminuye su ingreso. En consecuencia, el Informe sobre Desarrollo Humano (2019b) advierte que los niños nacidos en familias de ingreso bajo son más propensos a gozar de mala salud y a alcanzar niveles de estudios más bajos y, por ende, accederán, probablemente, a salarios más bajos, al tiempo que los niños con peor salud tienen mayor riesgo de no poder asistir a la escuela. Cuando los niños crecen, lo más seguro es que formen una pareja con otra persona de un estatus socioeconómico similar, y así las desigualdades pueden transmitirse de generación en generación.

Stiglitz (2012, p. 36):

“Los fallos de la política y la economía están interrelacionados, y se potencian mutuamente. Un sistema político que amplifica la voz de los ricos ofrece muchas posibilidades para que las leyes y la normativa -y su administración- se diseñen de forma que no solo no protejan a los ciudadanos corrientes frente a los ricos, sino que enriquezcan aún más a los ricos a expensas del resto de la sociedad".

Además, Bancaja e Ivie (2010, p. 3) afirman que el PIB per cápita no computa determinadas actividades realizadas fuera del mercado que son cruciales para el bienestar (cuidado de niños y ancianos).

La élite económica ha presionado para lograr un marco que le beneficia, a expensas de los demás, pero se trata de un sistema económico que no es ni eficiente ni justo. Con un sistema político que es tan sensible a los intereses económicos, el aumento de la desigualdad económica da lugar a un creciente desequilibrio en el poder político, y a una relación viciada entre política y economía (Stiglitz, 2012). Para Stiglitz, en cierto modo, la causa de ello es que gran parte de las desigualdades son consecuencia de las distorsiones del mercado, el cual tiene incentivos dirigidos no a crear nueva riqueza, sino a arrebatársela a los demás. Así pues, no es de extrañar que nuestro crecimiento haya sido mayor en los periodos en que la desigualdad ha sido menor y en los que hemos crecido todos juntos. Según Sevilla (2020, p. 1) la desigualdad no es algo natural, sino que requiere una justificación de las reglas que permitan una adquisición legítima de la propiedad por parte de un grupo o de un individuo.

Para Martínez et al. (2008) el crecimiento económico (entendido como el incremento del PIB) es necesario, pero no suficiente para mejorar las condiciones de vida en una sociedad. Bajo esta perspectiva se encuentran las aportaciones para el desarrollo endógeno de Becattini, Fuá, Garofoli, Stòhr, Vázquez Barquero y sobre el desarrollo humano Anand, Repetto, Sen, etc.

Bancaja e Ivie (2010, p. 3):

“El PIB puede incrementarse con el aumento de consumo de gasolina de una ciudad. También puede incrementarse como consecuencia de la delincuencia, al realizarse para combatirla un mayor gasto en seguridad. Por otro lado, detrás de una renta media elevada, puede haber profundas desigualdades en el reparto de ingresos, e incluso una extrema pobreza".

Tanto en el desarrollo endógeno como en el desarrollo humano se incorporan dos aspectos fundamentales que son la organización y la sostenibilidad. La organización retoma las relaciones e interacciones que existen entre los actores del sistema cuyo desarrollo se quiere analizar, y, por lo tanto, incluyen desde el funcionamiento del sector productivo hasta el de las instituciones de gobierno y de la sociedad civil. Para Martínez et al. (2008), la sostenibilidad se incorpora de forma que trasciende la visión acotada del uso racional y renovable de los recursos naturales, y se concibe como una visión integral de evolución de un sistema económico. En cambio, según 
Bancaja e Ivie (2010, p. 3) la contabilización del PIB puede crecer en el presente sacrificando bienestar futuro, ya sea sobreexplotando los recursos naturales o recurriendo al endeudamiento externo. El incremento del PIB per cápita puede no ir ligado a la mejora del bienestar social e incluso suponer retrocesos. Esquivel et al. $(2003$, p. 5) informan de que el impacto del desarrollo humano sobre el crecimiento será mayor mientras más grande sea la tasa de inversión en capacidades. Mientras la distribución de ingreso sea menos desigual, se puede alcanzar un círculo virtuoso que permita la elevación del nivel de calidad de vida de la población.

En la literatura económica, a partir del caso emblemático de Schultz (1961), Becker (1964) y Mincer (1974) quienes impulsan las ideas y conceptos de capital humano, se ha generalizado en informes técnicos sobre pobreza, bienestar y desarrollo humano, el enfoque de la educación como una inversión, donde ésta sería rentable en una economía de mercado o capitalista, siempre y cuando redituara en ingresos futuros más de lo que se invierte en educación (Scott, 2011). El cambio en la composición de la pobreza, en un país más educado, es producto entonces de problemas en la distribución; distribución de lo producido y las reglas bajo las que se realiza (Reyes \& López, 2017, pp. 51-54). Bajo esta lógica, para Govea (2018) es en el capital humano donde deben dirigirse las inversiones de un país.

A nivel mundial, aunque los valores del IDH han aumentado en todas las regiones y grupos de desarrollo humano, los porcentajes varían considerablemente. Asia Meridional fue la región que creció más rápido entre 1990 y 2017, con un 45,3\%, seguida por Asia Oriental y el Pacifico, con un $41,8 \%$, y África Subsahariana, con un 34,9\%. En cambio, los países de la Organización para la Cooperación y el Desarrollo Económicos (OCDE) crecieron un 14,0\% (Arrieta, 2018). Los cinco países con el más alto índice son: Noruega $(0,954)$, Suiza $(0,946)$, Irlanda $(0,942)$, Alemania $(0,939)$, Australia $(0,938)$, y los que ocupan los últimos cinco puestos son Burundi $(0,426)$, Sudán del Sur $(0,413)$, Chad $(0,401)$, República Centroafricana $(0,381)$ y Níger $(0,377)$ (Organización de las Naciones Unidas, 2018).

Las desigualdades en algunas capacidades básicas se van reduciendo lentamente en la mayoría de los países, aunque todavía queda mucho camino por recorrer. La esperanza de vida al nacer, el porcentaje de población con estudios primarios y el número de suscripciones de telefonía móvil muestran una reducción de las desigualdades en todos los grupos de desarrollo humano (PNUD, 2019, p. 8). Es posible invertir las tendencias de la desigualdad; otros países lo han conseguido. Brasil tenía uno de los niveles más altos de desigualdad del mundo, pero durante la década de los noventa se dio cuenta de los peligros, en términos tanto de su potencial de división social y política como de crecimiento económico a largo plazo. El resultado fue un consenso político a lo largo de toda la sociedad de que había que hacer algo. Bajo el mandato del presidente Henrique Cardoso hubo un aumento masivo del gasto en educación, incluida la destinada a los más pobres. Con el presidente Luiz Inácio Lula da Silva hubo gastos sociales para reducir el hambre y la pobreza; se redujo la desigualdad, aumentó el crecimiento y la sociedad se hizo más estable.

Stiglitz (2012, p. 57):

"Brasil sigue teniendo más desigualdad que Estados Unidos, pero mientras que Brasil ha luchado, con bastante éxito, para mejorar las condiciones de vida de los pobres y reducir las diferencias de renta entre ricos y pobres, Estados Unidos ha permitido que crezca la desigualdad y aumente la pobreza".

Salazar \& García (2014, p. 14):

"Los determinantes sociales de la salud son las circunstancias en que nacen, crecen, viven, trabajan y envejecen las personas. Según la OMS estas circunstancias son resultado de la distribución del dinero, el poder, los recursos a nivel mundial, nacional y local que depende de las políticas adoptadas". 
Según el Índice de Indicadores de desarrollo humano (2018) gran parte de la discusión sobre la desigualdad de ingresos se centra en el ingreso y la riqueza del $1 \%$ más rico de la población de cada país e incluso del 0,1\% más rico en relación con el resto. Los informes recientes de Oxfam International muestran que 8 personas poseen ya la misma riqueza que 3.600 millones de personas, la mitad más pobre de la humanidad; y que el $82 \%$ del crecimiento de la riqueza mundial del último año ha ido a parar a manos del 1\% más rico, mientras que la mitad más pobre de la población mundial no ha recibido nada de ese crecimiento.

\subsection{Correlación Índice de Desarrollo Humano y Coeficiente de Gini}

En cuanto al IDH, el informe sobre el Índice e Indicadores de Desarrollo Humano (Organización de las Naciones Unidas, 2018) considera que los países que alcanzan un grado de 0,80 poseen un Índice de Desarrollo Humano calificado como "muy alto"; en 2018 se estimaba que 58 países alcanzaban este rango. Se conoce como país subdesarrollado, o país en vías de desarrollo, a aquel que tiene un Producto Interno Bruto per cápita, un Producto Nacional Bruto (PNB) per cápita y un IDH bajos en relación con otros países más desarrollados. El subdesarrollo de estos países no se refiere simplemente a que se encuentren en una etapa previa al desarrollo; que un país sea subdesarrollado significa que, en ese momento particular, "dicho país tiene una situación que no le permite alcanzar mejores estándares de calidad de vida y económicos" (Arrieta, 2018, p. 1), el Programa de las Naciones Unidas para el Desarrollo considera que los países que alcanzan un grado entre 0,50 y 0,79, poseen un Índice de "medio" o "alto". En 2018, se estimaba que 92 países se encontraban dentro de este rango (Arrieta, 2018).

Los países menos desarrollados son aquellos, señala Arrieta, que presentan problemas estructurales de tal magnitud, que impiden su desarrollo socioeconómico y que son altamente vulnerables a catástrofes económicas y ambientales. Estas son naciones subdesarrolladas que presentan un grado de desarrollo muy bajo, cuentan con el PIB per cápita, el PNB per cápita y el IDH más bajo a nivel mundial. La Organización de las Naciones Unidas clasifica a 47 países como menos desarrollados.

En las siguientes gráficas se presenta el Índice de Desarrollo Humano y el Coeficiente de Gini del año 1990, para un grupo de países tanto desarrollados como no desarrollados, a fin de observar en qué medida los países con mayor desarrollo son los que tienen un coeficiente menor.

Figura 1. Índice de Desarrollo Humano 1990.

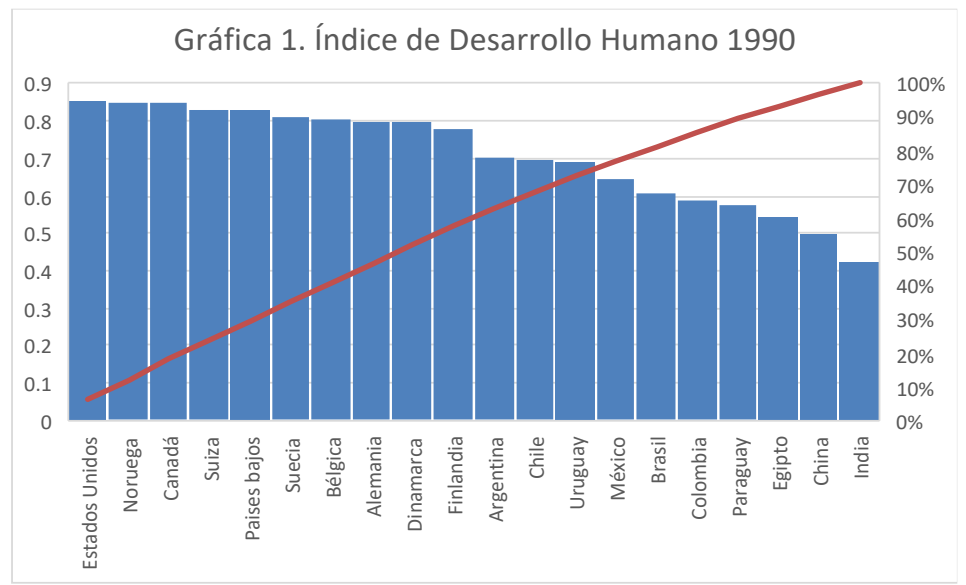

Fuente: Organización de las Naciones Unidas (2020) y Economipedia (2019). 
Figura 2. Coeficiente de Gini 1990.

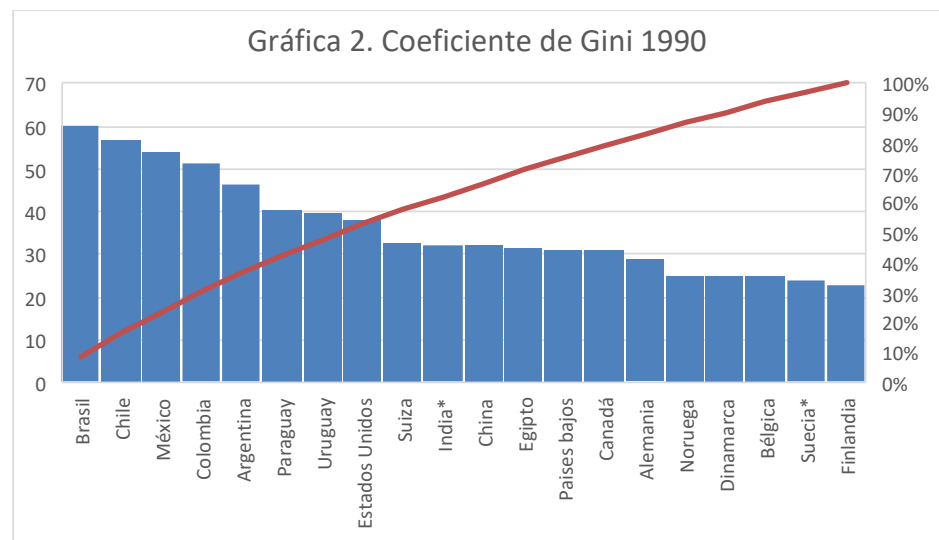

Fuente: Banco Mundial (2020) y Knoema (2019).

Para el 2018, el comportamiento de ambas variables es el siguiente:

Figura 3. Índice de Desarrollo Humano 2018.

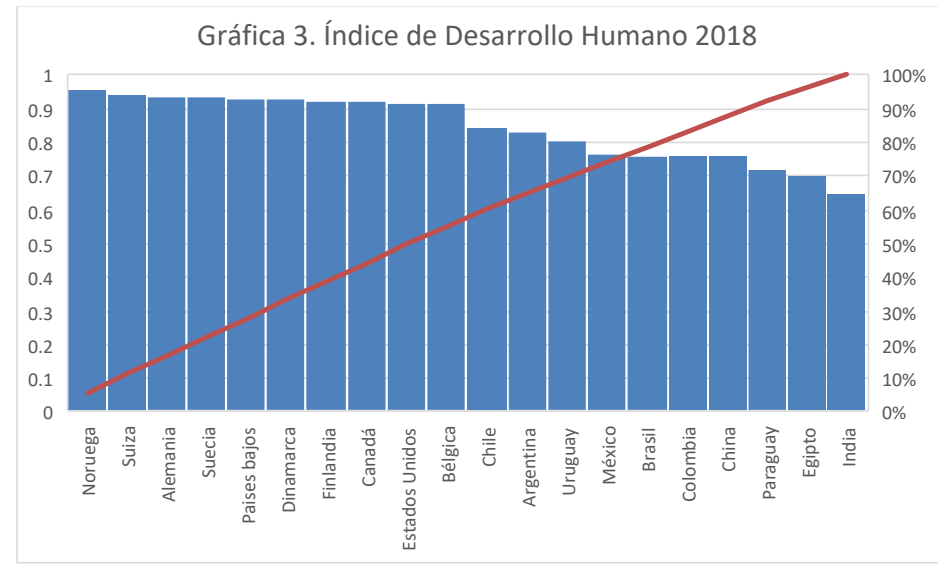

Fuente: Organización de las Naciones Unidas (2020) y Economipedia (2019).

De igual forma, para el Coeficiente de Gini es el que se muestra a continuación:

Figura 4. Coeficiente de Gini 2018.

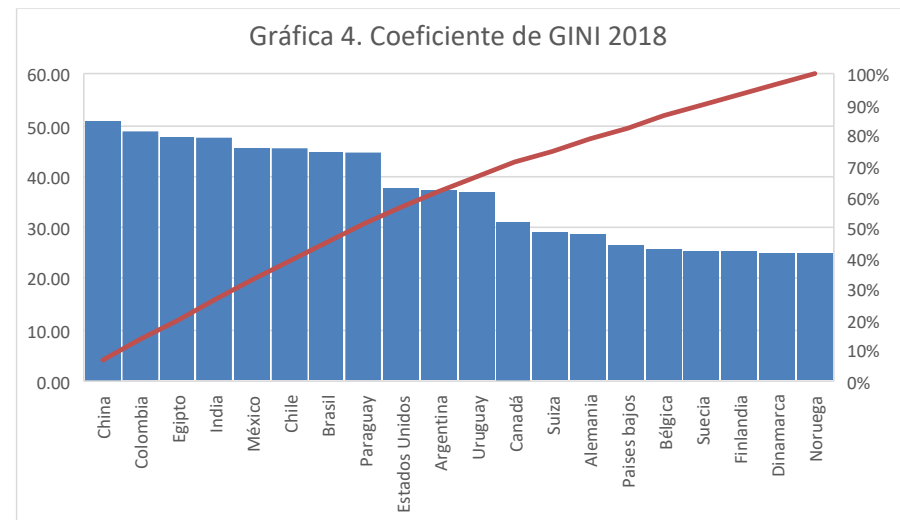

Fuente: Banco Mundial (2020) y Knoema (2019). 
Si observamos el comportamiento de estas dos variables en cada uno de los años estudiados, nos damos cuenta de que, efectivamente, los países con mayor desarrollo humano tienen un Coeficiente de Gini menor, particularmente en el año 2018, lo que nos lleva a suponer que si existe una correlación entre estas dos variables (IDH y CG), y que dicha relación es negativa: mayor desarrollo humano, menor desigualdad. Lo que nos lleva a suponer que, en los últimos años las naciones que mejoraron los niveles de bienestar lo hicieron distribuyendo también el ingreso.

\section{Materiales, métodos y resultados}

A fin de identificar la relación que guardan el Índice de Desarrollo Humano y el Coeficiente de Gini se realizó una gráfica de dispersión para los dos años analizados, en ambas graficas se observa una relación inversa entre las dos variables.

Figura 5. IDH y Coeficiente de Gini 1990.

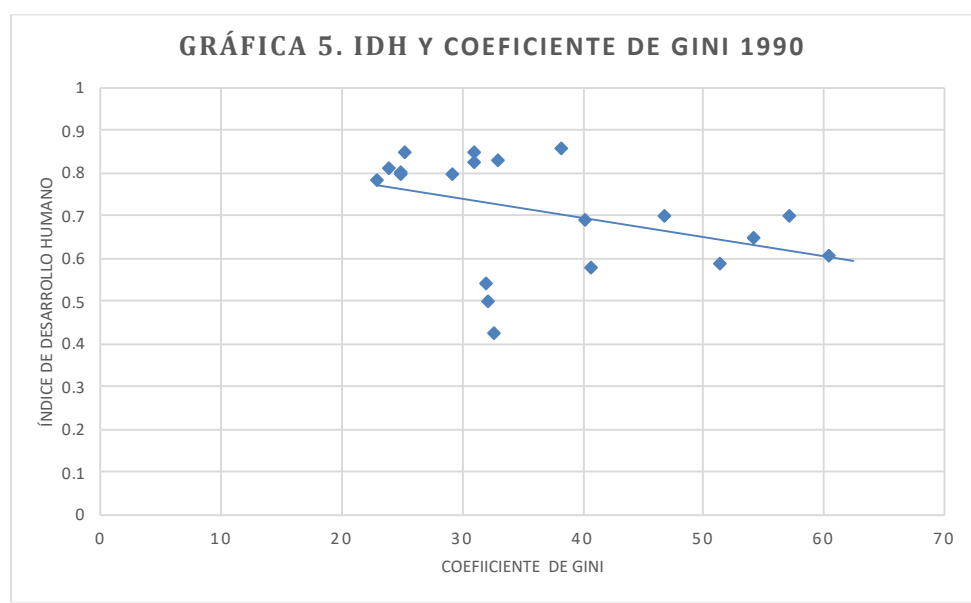

Fuente: Banco Mundial (2020) y Organización de las Naciones Unidas (2020).

Para el 2018, se observa una menor dispersión, tal y como muestra la figura 6.

Figura 6. IDH y Coeficiente de Gini 2018.

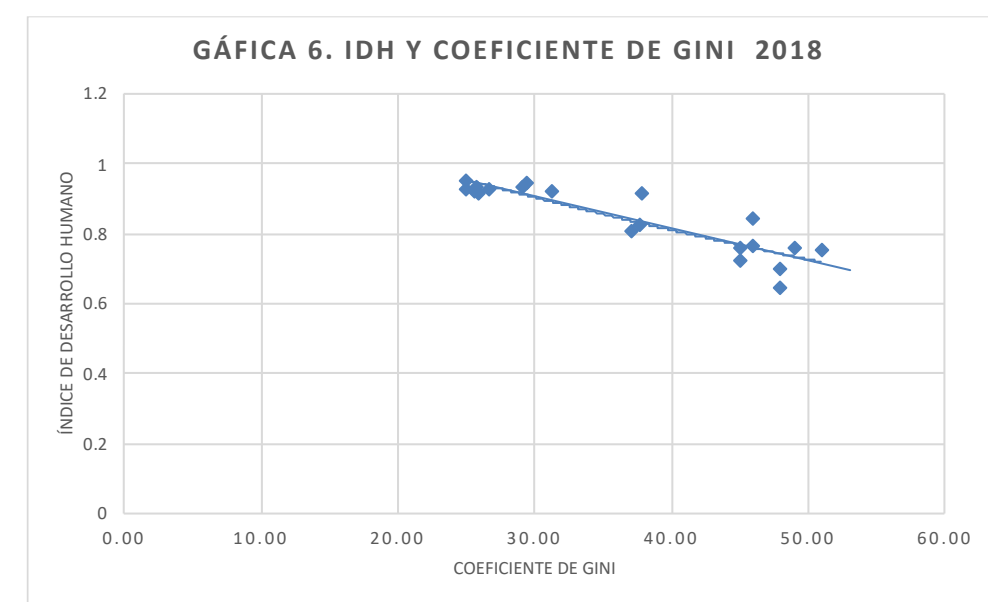

Fuente: Banco Mundial (2020) y Organización de las Naciones Unidas (2020). 


\subsection{Correlación Bivariada}

Tratando de determinar y cuantificar la relación que mantienen las variables estudiadas, se calculó la correlación bivariada. Esta es una herramienta útil para medir la fuerza y relación entre dos variables, su resultado nos muestra si la relación es fuerte o débil, directa o inversamente proporcional. Si las dos variables se mueven en la misma dirección la correlación es positiva y si lo hacen en relación opuesta es negativa: no implica causalidad. Se afirma que hay correlación de variables si al moverse los valores de la variable A, también lo hacen los de la variable B. Así pues, la correlación puede variar de intensidad y dirección.

En el análisis de correlación de variables existen tres coeficientes comúnmente utilizados y son: el de Pearson, el de Kendall y el de Spearman, todos basados en la misma información, aunque usan fórmulas diferentes. La estimación de uno u otro depende de la naturaleza de las variables a analizar, si es cualitativa o cuantitativa (Morales \& Rodríguez, 2016).

La correlación de Pearson evalúa la relación lineal entre dos variables continuas, una relación es lineal cuando un cambio en una variable se asocia con un cambio proporcional en la otra variable, este método es más adecuado cuando las variables siguen la curva normal. Es una buena alternativa cuando las variables se encuentran en un nivel de medición de intervalos, pero no se puede cumplir satisfactoriamente con los requisitos básicos para el uso de una prueba paramétrica. Así, es la que se usó en la presente investigación.

En este estudio se realizaron dos correlaciones una para el año 1990 y otra para el año 2018, ambas para las variables IDH y Coeficiente de Gini. Como podemos apreciar en ambos casos, la correlación es significativa y negativa. Lo que nos indica que, si una variable se mueve, también lo hará la otra de manera inversa: si una aumenta la otra disminuye.

No obstante, el nivel de correlación es menor en el año 1990, como lo podemos observar en las tablas 1 y 2 .

Tabla 1. Correlaciones IDH-Coeficiente de Gini 1990.

\begin{tabular}{llr|r}
\multicolumn{2}{c}{ Correlaciones IDH-C.GINI 1990 } & \\
& & COEFICIENTE \\
& & IDH 1990 & DE GINI 1990 \\
\hline Correlación de & IDH 1990 & 1,000 &,- 399 \\
Pearson & COEFICIENTE DE GINI 1990 &,- 399 & 1,000 \\
\hline Sig. (unilateral) & IDH 1990 &. &, 041 \\
\cline { 2 - 4 } & COEFICIENTE DE GINI 1990 &, 041 &. \\
\hline N & IDH 1990 & 20 & 20 \\
\hline & COEFICIENTE DE GINI 1990 & 20 & 20 \\
\hline
\end{tabular}

Fuente: Elaboración propia (2020).

Para el 2018 la correlación es igualmente negativa pero mucho más alta, casi cercana a uno. 
Tabla 2. Correlaciones IDH-Coeficiente de Gini 2018.

\section{Correlaciones IDH-C.GINI 2018}

\begin{tabular}{llr|r} 
& \multicolumn{2}{c}{ Correlaciones IDH-C.GINI 2018 } & \multicolumn{1}{c}{ COEFICIENTE } \\
& & IDH 2018 & DE GINI 2018 \\
\hline Correlación de & IDH 2018 & 1,000 &,- 896 \\
\cline { 2 - 4 } Pearson & COEFICIENTE DE GINI 2018 &,- 896 & $\mathbf{1 , 0 0 0}$ \\
\hline Sig. (unilateral) & IDH 2018 &. &, 000 \\
\cline { 2 - 4 } & COEFICIENTE DE GINI 2018 &, 000 &. \\
\hline N & IDH 2018 & 20 & 20 \\
\hline
\end{tabular}

Fuente: Elaboración propia (2020).

\subsection{Regresión Lineal}

Una vez que determinamos que, si hay correlación entre las dos variables analizadas, buscamos cuantificar algún grado de dependencia entre ellas, entendiendo que existen más variables y que, por tanto, el modelo no podría ser totalmente fiable. Aunque Schmidt (2005) señala que el análisis de regresión nos indica que dos variables tienen una relación matemática y nada nos dice sobre la causa, consideramos que sí nos muestra una tendencia general de causalidad.

Así pues, con el fin de identificar si hay algún tipo dependencia entre el Índice de Desarrollo Humano y el Coeficiente de Gini, el primero como dependiente del segundo, se realizó el análisis de regresión lineal simple, obteniendo los siguientes resultados.

Tabla 3. Resumen del modelo 1990.

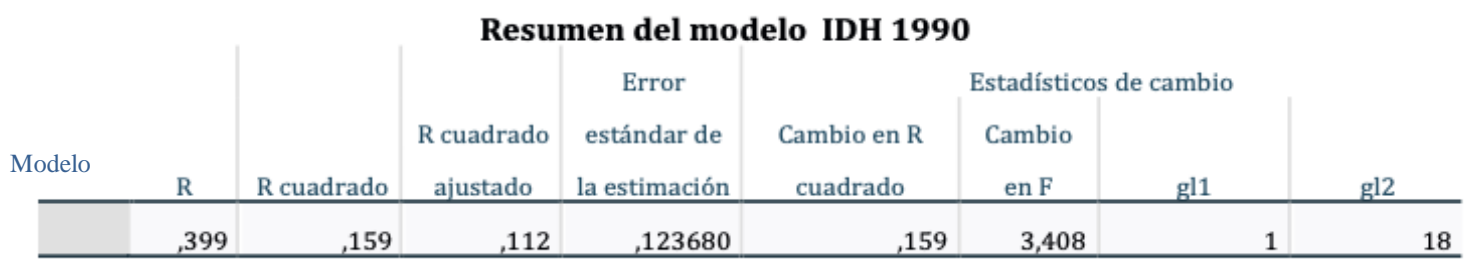

Resumen del modelo 1990

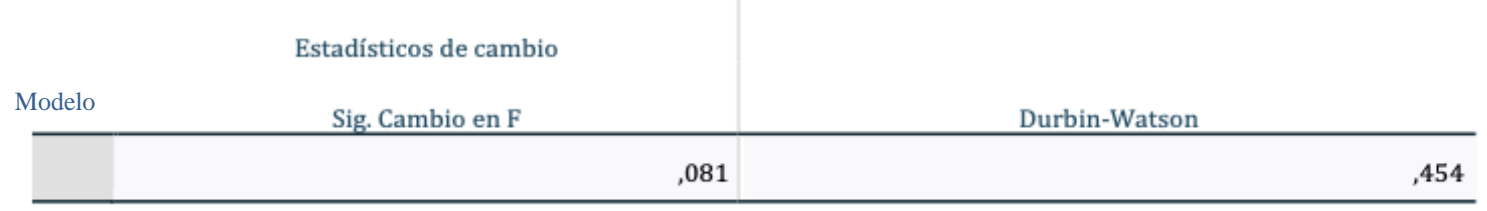

Fuente: Elaboración propia (2020).

Casi 30 años después, el modelo arrojó un valor de $R$ mucho mayor, como ya se advirtió en el coeficiente de Pearson, lo que nos lleva a suponer que, en los últimos años, los países que han aumentado su desarrollo lo han hecho redistribuyendo el ingreso nacional. 
Tabla 4. Resumen del modelo 2018.

\begin{tabular}{|c|c|c|c|c|c|c|c|c|}
\hline \multirow[b]{3}{*}{ Modelo } & \multirow[b]{3}{*}{$\mathrm{R}$} & \multicolumn{6}{|c|}{ Resumen del modelo IDH 2018} & \multirow[b]{3}{*}{$\mathrm{g} 12$} \\
\hline & & & & Error & & stadísticos de & mbio & \\
\hline & & R cuadrado & $\begin{array}{c}\text { R cuadrado } \\
\text { ajustado }\end{array}$ & $\begin{array}{c}\text { estándar de } \\
\text { la estimación }\end{array}$ & $\begin{array}{c}\text { Cambio en } \mathrm{R} \\
\text { cuadrado }\end{array}$ & $\begin{array}{c}\text { Cambio en } \\
\text { F }\end{array}$ & gl1 & \\
\hline 1 & 896 & ,803 & 793 & 044576 & ,803 & 73,580 & 1 & 18 \\
\hline
\end{tabular}

Resumen del modelo 2018

Estadísticos de cambio

\begin{tabular}{lr|r} 
Modelo & Sig. Cambio en F & Durbin-Watson \\
\hline 1 &, 000 & 1,689 \\
\hline
\end{tabular}

Fuente: Elaboración propia (2020).

Los coeficientes del IDH $\left(\beta_{0}\right)$ y del Coeficiente de Gini $\left(\beta_{1}\right)$ para cada año fueron los siguientes:

Tabla 5. Coeficientes IDH-Gini 1990.

\begin{tabular}{|c|c|c|c|c|c|c|}
\hline \multirow{3}{*}{ Modelo } & \multicolumn{5}{|c|}{ Coeficientes IDH-C.GINI 1990} & \multirow{3}{*}{$\begin{array}{c}95.0 \% \\
\text { intervalo de } \\
\text { confianza para } \\
\text { B } \\
\text { Límite inferior }\end{array}$} \\
\hline & \multicolumn{2}{|c|}{$\begin{array}{l}\text { Coeficientes no } \\
\text { estandarizados }\end{array}$} & \multirow{2}{*}{$\begin{array}{c}\text { Coeficientes } \\
\text { estandarizados } \\
\text { Beta } \\
\end{array}$} & \multirow[b]{2}{*}{$\mathrm{t}$} & \multirow[b]{2}{*}{ Sig. } & \\
\hline & B & Desv. Error & & & & \\
\hline (Constante) &, 876 & 093 & & 9,408 & 000 &, 680 \\
\hline $\begin{array}{l}\text { COEFICIEN } \\
\text { TE DE GINI } \\
1990\end{array}$ &,- 004 & 002 &,- 399 & $-1,846$ & 081 &,- 010 \\
\hline
\end{tabular}

\begin{tabular}{|c|c|c|c|c|c|c|}
\hline \multirow[b]{2}{*}{ Modelo } & \multirow{2}{*}{$\begin{array}{c}95.0 \% \\
\text { intervalo de } \\
\text { confianza } \\
\text { para B } \\
\text { Límite } \\
\text { superior } \\
\end{array}$} & \multicolumn{3}{|c|}{ Correlaciones } & \multicolumn{2}{|c|}{$\begin{array}{l}\text { Estadísticas de } \\
\text { colinealidad }\end{array}$} \\
\hline & & $\begin{array}{c}\text { Orden } \\
\text { cero }\end{array}$ & Parcial & Parte & Tolerancia & VIF \\
\hline (Constante) & 1,071 & & & & & \\
\hline $\begin{array}{l}\text { COEFICIENTE DE } \\
\text { GINI } 1990\end{array}$ & ,001 &,- 399 &,- 399 &,- 399 & 1,000 & 1,000 \\
\hline
\end{tabular}

Fuente: Elaboración propia (2020). 
Realizamos un análisis tras 28 años después, el resultado es el siguiente:

Tabla 6. Coeficientes IDH-Gini 1990.

\begin{tabular}{|c|c|c|c|c|c|c|}
\hline \multirow{3}{*}{ Modelo } & \multicolumn{6}{|c|}{ Coeficientes IDH-C.GINI 2018} \\
\hline & \multicolumn{2}{|c|}{$\begin{array}{l}\text { Coeficientes no } \\
\text { estandarizados }\end{array}$} & $\begin{array}{c}\text { Coeficiente } \\
\text { s } \\
\text { estandariza } \\
\text { dos }\end{array}$ & & & $\begin{array}{c}95.0 \% \\
\text { intervalo de } \\
\text { confianza } \\
\text { para B }\end{array}$ \\
\hline & $\mathrm{B}$ & Desv. Error & Beta & $\mathrm{t}$ & Sig. & inferior \\
\hline (Constante) & 1,177 & 040 & & 29,565 & 000 & 1,093 \\
\hline $\begin{array}{l}\text { COEFICIENTE DE GINI } \\
2018\end{array}$ &,- 009 & 001 &,- 896 & $-8,578$ & 000 &,- 011 \\
\hline
\end{tabular}

\begin{tabular}{|c|c|c|c|c|c|c|}
\hline \multirow{2}{*}{ Modelo } & \multirow{2}{*}{$\begin{array}{c}95.0 \% \\
\text { intervalo de } \\
\text { confianza } \\
\text { para B } \\
\text { Límite } \\
\text { superior } \\
\end{array}$} & \multicolumn{3}{|c|}{ Correlaciones } & \multicolumn{2}{|c|}{$\begin{array}{l}\text { Estadísticas de } \\
\text { colinealidad }\end{array}$} \\
\hline & & Orden cero & Parcial & Parte & Tolerancia & VIF \\
\hline (Constante) & 1,261 & & & & & \\
\hline $\begin{array}{l}\text { COEFICIENTE DE GINI } \\
2018\end{array}$ &,- 007 &,- 896 &,- 896 &,- 896 & 1,000 & 1,000 \\
\hline
\end{tabular}

Fuente: Elaboración propia (2020).

Basados en el anterior resultado, la ecuación matemática estimada para cada año es:

$$
I D H=\beta_{0}+\beta_{1} \text { C. Gini } \quad \text { (Ecuación 1) }
$$

Esta ecuación establece que el IDH es igual al coeficiente $\beta_{0}$ (la constante) si el coeficiente Gini no cambia, y el coeficiente $\beta_{1}$, varia por cada unidad que se modifique el C. Gini considerando que existen muchos otros factores que influyen y determinan el IDH se incluyó el termino de error $\varepsilon_{i}$, que representa los efectos en el proceso de generación de datos no contemplados en la relación lineal, resultando la siguiente ecuación:

$$
I D H=\beta_{0}+\beta_{1} \text { C. Gini }+\varepsilon_{i} \quad(\text { Ecuación 2) }
$$

La ecuación estimada se expresa:

$$
I D H=\hat{\beta}_{0}+\hat{\beta}_{1} \text { C.Gini }+\hat{\varepsilon}_{i} \quad(\text { Ecuación } 3)
$$

Sustituyendo los valores de los coeficientes, tenemos las siguientes ecuaciones matemáticas:

Para 1990:

$$
\left.I D H_{1990}=0.876-.004 C . \text { Gini }+\hat{\varepsilon}_{i} \quad \text { (Ecuación } 4\right)
$$


Para 2018:

$$
I D H_{2018}=1.177-.009 \text { C. Gini }+\hat{\varepsilon}_{i} \quad(\text { Ecuación } 5)
$$

Como podemos observar el valor de $\beta_{1}$, en ambos casos es negativo, lo que nos ratifica los resultados que muestran las dos gráficas de dispersión antes señaladas.

En este caso $\hat{\varepsilon}_{i}$ es el residual estimado "el residual de un punto de datos es la distancia entre él y la línea estimada" (Schmidt, 2005, p. 83). La ecuación del residual quedo:

$$
\hat{\varepsilon}_{i}=I D H-\beta_{0}-\beta_{1} C . \text { Gini } \quad \text { (Ecuación 6) }
$$

El Residual para cada año es:

$$
\begin{aligned}
& \hat{\varepsilon}_{i}=I D H_{1990}-0.876+.004 \text { C. Gini } \quad \text { (Ecuación 7, 1990) } \\
& \hat{\varepsilon}_{i}=I D H_{2018}-1.177+.009 \text { C. Gini } \quad \text { (Ecuación 8, 2018) }
\end{aligned}
$$

Entonces, según ambas ecuaciones podemos inferir que, un aumento del valor de C. Gini provocará una disminución del Índice de Desarrollo Humano y a la inversa una disminución del Coeficiente de Gini aumentará el desarrollo humano.

De esta forma, para disminuir la desigualdad, según Keynes (Sevilla, 2020) el gobierno debe introducir importantes correcciones al disfrute privado de la propiedad a través de impuestos progresivos para financiar políticas sociales. Esto es, quitar a quienes más tienen (disminuir la desigualdad) y dar a quienes no tienen o tienen poco a través de la creación de escuelas, hospitales, vivienda, servicios públicos, etc. (aumentar el bienestar).

Sevilla (2020):

"Compensar con medidas redistributivas la tendencia natural del capitalismo, poner límites a la propiedad privada que abre el espacio a esa igualdad de oportunidades que debería activar el ascensor social para que ningún individuo quedará atrapado en una desigualdad heredada por la clase social de la familia en la que nace "(p.1).

\section{Conclusiones}

El aumento del PIB no es suficiente para que un país tenga desarrollo humano alto y logre disminuir los niveles de desigualdad. Entonces, ¿Qué es lo que hace falta? Una mejor distribución de éste, a través de una serie de políticas públicas redistributivas. La disminución de la desigualdad es una cuestión multidimensional y, por ello, debe de ir acompañada de políticas públicas trasversales.

Por lo tanto, si los gobiernos quieren aumentar el Índice de Desarrollo Humano y disminuir las brechas de desigualdad, deben poner mayor énfasis en la redistribución de la riqueza nacional. Un mecanismo, comúnmente utilizado, son las políticas fiscales, en particular una política impositiva progresiva: los impuestos (ya sean sobre los ingresos, el patrimonio o el consumo) pueden ser muy útiles para corregir las desigualdades, ya que éstos permiten recaudar ingresos para mejorar los servicios de salud, vivienda, educación y proporcionar un seguro social que beneficia tanto a las personas pobres como a quienes se encuentran en el tramo intermedio de la distribución de los ingresos, además de una serie de servicios públicos en general. 


\section{Referencias}

Arrieta, E. (2018). Países Desarrollados y países subdesarrollados [post en blog]. Diferenciador. Disponible en https://www.diferenciador.com/paises-desarrollados-y-paises-subdesarrollados/

Bancaja e Ivie (2010). Capital humano, Desarrollo humano, Compromiso Social. Fundación Bancaja e Ivie, cuaderno 114, España.

Banco Mundial (2020). Índice de Gini [Banco de datos]. Disponible en: https://datos.bancomundial.org/indicator/SI.POV.GINI

Bustos Serrano, F.J. (2018). La desigualdad según Piketty [post en blog]. El Captor. Disponible en: http://www.elcaptor.com

Economipedia (2019). Ranking Índice de Desarrollo Humano [Banco de datos]. Disponible en: https://economipedia.com/ranking/ranking-indice-de-desarrollo-humano-2018.html

Esquivel, G., López, L.F. y Vélez, G. R. (2003). Crecimiento económico, desarrollo humano y desigualdad regional en México 1950-2000. Estudio sobre Desarrollo Humano, PNUD México.

Govea Maridueña, A. (2018). Evolución e impacto del PIB y el IDH en un mundo desigual. Revista Vinculando. Disponible en: http://vinculando.org/sociedadcivil/evolucion-e-impacto-pib-idh-mundodesigual.html

Knoema (2019). Índice Gini [Banco de datos]. Disponible en: https://knoema.es/atlas/topics/Pobreza/Desigualdad-del-ingreso/\%C3\%8Dndice-GINI

Lizárraga, C. (2013). El Índice de Gini: la desigualdad a la palestra. Revista eXtoikos, 10, 67-69.

Manfred, N. (2014). PIB, Desarrollo Humano y Bienestar [post en blog]. Cristianisme y Justicia. Disponible en: https://blog.cristianismeijusticia.net/2014/09/19/pib-desarrollo-humano-y-bienestar

Martínez, P. S., Flamand, L. y Hernández, A. (2008). Panorama del desarrollo Municipal en México, Antecedentes, diseño y hallazgos Índice de Desarrollo Municipal Básico. Revista Gestión y Política Pública, $17(1), 145-192$.

Morales, P., y Rodríguez, L. (2016). Aplicación de los coeficientes correlación de Kendall y Spearman. Universidad Nacional Experimental de los Llanos Occidentales "Ezequiel Zamora".

Organización de las Naciones Unidas (2018). Índice e indicadores de desarrollo humano, actualización estadística 2018 [Banco de datos].

Organización de las Naciones Unidas (2019a). Transformando México desde lo local. Informe de Desarrollo Humano Municipal 2010-2015. México.

Organización de las Naciones Unidas (2019b). Mas allá del ingreso, más allá de los promedios, más alá del presente: Desigualdades de desarrollo humano en el siglo XXI. Panorama General, Informe sobre Desarrollo Humano 2019.

Organización de las Naciones Unidas (2020). Human Development Index (HDI) [Banco de datos]. Disponible en: http://hdr.undp.org/en/indicators/137506\# 
Picazzo, E., Gutiérrez, E., Infante, J. M., y Cantú, P. C. (2011). La teoría del desarrollo humano y sustentable: hacia el reforzamiento de la salud como un derecho y libertad universal. Estudios sociales, 19(37), 253-279.

Piketty, T. (2020). Tomas Piketty: Máxima desigualdad de la riqueza, máxima desigualdad de la riqueza [post en blog]. El Economista. Disponible en: https://www.eleconomista.com.mx/opinion/Thomas-PikettyMaxima-desigualdad-de-la-riqueza-maxima-desigualdad-del-ingreso-20200301-0082.html

Reyes, M. y López, M. (2017). El bienestar y la pobreza en México. Tendencias de los mercados laborales desde una perspectiva de derechos humanos. Observatorio de Salarios, Universidad Iberoamericana Puebla.

Salazar, R. E., y García, J. M. (2014). El Índice de Desarrollo Humano como indicador social. Nómadas. Critical Journal of Social and Juridical Sciences, 44(4).

Schmidt, J. (2005). Econometría. McGraw-Hill.

Scott, J. (2011). Gasto público y desarrollo humano en México: análisis de incidencia y equidad. Documento de trabajo para el informe sobre desarrollo humano México.

Sen, A. (2000). El desarrollo como libertad. Gaceta ecológica, 55, 14-20.

Sevilla, J. (2020). Las propuestas de Tomas Piketty contra la desigualdad [post en blog]. Nueva Revista. Disponible en: https://www.nuevarevista.net/destacados/las-propuestas-de-thomas-piketty-contra-ladesigualdad/

Stiglitz, J. E. (2012). El precio de la desigualdad: el 1\% de población tiene lo que el 99\% necesita. Taurus.

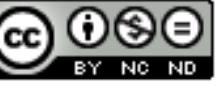

(C) Attribution-NonCommercial-NoDerivatives 4.0 International (CC BY-NC-ND 4.0)

https://creativecommons.org/licenses/by-nc-nd/4.0/ 